\title{
La fabrique de Paris au XIX siècle : qualités de produits et formations des fabricants
}

\section{Nadège Sougy}

\section{(2) OpenEdition}

1 Journals

\section{Édition électronique}

URL : https://journals.openedition.org/artefact/9374

DOI : 10.4000/artefact.9374

ISSN : 2606-9245

Éditeur :

Association Artefact. Techniques histoire et sciences humaines, Presses universitaires du Midi

\section{Édition imprimée}

Date de publication : 11 septembre 2014

Pagination : 89-99

ISBN : 978-2-271-08150-6

ISSN : 2273-0753

Référence électronique

Nadège Sougy, "La fabrique de Paris au xIx siècle : qualités de produits et formations

des fabricants ", Artefact [En ligne], 2 | 2014, mis en ligne le 12 mai 2021, consulté le 24 août 2021.

URL : http://journals.openedition.org/artefact/9374; DOI : https://doi.org/10.4000/artefact.9374

\section{(c) (i) (9)}

Artefact, Techniques, histoire et sciences humaines est mise à disposition selon les termes de la Licence Creative Commons Attribution - Pas d'Utilisation Commerciale - Pas de Modification 4.0 International. 


\section{La fabrique de Paris au $x_{1 x}{ }^{e}$ siècle : qualités de produits et formations des fabricants}

Nadège Sougr ${ }^{1}$

\section{Résumé}

La réputation de la mode parisienne est-elle liée à la compétence de ses ouvriers? À la fin du xIX ${ }^{\mathrm{e}}$ siècle, alors que la fabrique parisienne est en crise, la question de la formation des ouvriers se pose. Cet article étudie la fabrication et la qualité des articles de Paris. En analysant les discours et les pratiques des fabricants, cette contribution révise l'importance des savoir-faire d'un secteur caractérisé par une forte division du travail entre des acteurs peu qualifiés mais dont la réputation repose sur le bon goût parisien.

Mots-clés : article de Paris, compétence, division du travail, qualité, réputation.

\section{Abstract}

Is the reputation of Parisian fashion imputable to the skills of its labor force?

At the end of the 19th century, when the French makers were facing economical turmoil, workers education became a critical issue. This paper investigates the manufacturing process and the notion of quality of Parisian fancy goods. The article reviews the significance of technical know-how through in-depth discourse and business practices analysis of the sector. This industry appears to be characterized by a strong division of work among weakly qualified workers and by its reputation mainly relying on Parisian good taste.

Keywords : division of labor, fancy goods, quality, reputation, skill. 
S'intéresser à la fabrique de Paris et, à travers elle, aux «articles de Paris» pour interroger le lien entre la qualité des produits et la formation des ouvriers peut sembler être une gageure. Derrière ce terme générique sont regroupées quantités de fabrications très variées qui n'autorisent que très difficilement une approche par la qualité, d'autant qu'elle semble interdire l'examen des formations, des compétences requises pour leur production. Or, selon les sources - enquête de la Chambre de commerce, expositions nationales ou universelles -, la classification «articles de Paris » est une rubrique hétéroclite, parfois fourre-tout qui, à défaut de réunir toutes les fabrications parisiennes, comprend celles qui ne semblent pas relever $d^{\prime}$ autres groupes et qui ne trouvent pas une place évidente ailleurs.

Si l'on se reporte au premier numéro de l'hebdomadaire La Fabrique de Paris. Moniteur du commissionnaire et de l'exportateur, publié en 1862, la définition n'apparaît pas plus claire: «Multiple, vague, indéfinie comme la fantaisie, le caprice et le goût qu'elle personnifie, elle a échappé jusqu'ici dans son ensemble à toutes les explorations. Nous mêmes qui prenons ici la tâche de grouper, de réunir les éléments dont elle se compose, nous ne sommes pas bien sûrs de ne rien omettre $^{2}$.»

Néanmoins, cette diversité trahit des points communs qui donnent corps à la catégorie. Il s'agit de fabrications relevant d'une grande division du travail, parisien ou non, et se distinguant par «le Goût et par une recherche incessante de la nouveauté ${ }^{3}$. Dès lors, la fabrique de Paris apparaît comme un terrain d'expertise spécifique pour saisir comment se trouve posé ou non le lien entre qualité de produits et formation des agents engagés dans ces fabrications. Par qualité, il faut écarter l'approche classique de bonne ou de mauvaise qualité, pour retenir celle qui en fait une variable d'ajustement reposant sur les caractéristiques esthétiques et techniques du produit conditionnées par les intérêts d'un producteur, et dont la pertinence est liée à son acceptation par le marché. Mais la qualité procède également d'un discours qui construit une réputation sur le marché. Aussi est-il indispensable de comprendre ce que les fabricants, marchands et clients désignent sous le terme de qualité relativement aux articles dits «de Paris» qui se forgent dans ce que nous nommons une «identité de place» où la capitale est une référence sur le marché.

Rapporté à la compétence des fabricants ${ }^{4}$, il s'agit de comprendre la maîtrise d'un art dont la certification ne va pas de soi et semble être une préoccupation essentielle dès qu'il s'agit de signaler la qualité, le goût des articles de Paris face à la montée de la concurrence. Derrière la question de la qualité et de la formation, nous souhaitons saisir l'articulation à Paris de ce qui constitue une «identité de place», construite sur le goût de ses articles et sur celui de ses ouvriers. 


\section{La fabrique du goût}

\section{Un foisonnement hétéroclite}

Instruments d'astronomie, de physique, de chimie, de chirurgie, bijouterie en or, en doublé, en faux, en camé à la mécanique, rubis, émeraudes, topazes d'imitation, bronzes d'art, bronze de commerce, tabletterie, meubles de luxe, ébénisterie, coffrets sculptés et incrustés, articles d'étagères, bimbeloterie, papiers peints, papeterie de bureau, maroquinerie, reliures, gravures, estampes, articles de modes, de parures, fleurs artificielles, ganterie, parfumerie, éventails et objets liturgiques forment le gros des articles de Paris qui inondent le marché parisien pendant la huitaine entre Noël et le Jour de l'an. Relevant de secteurs industriels occupant $10 \%$ des ouvriers parisiens, la fabrication d'articles de Paris représente pour près de $4 \%$ du chiffre d'affaires total de Paris et $26 \%$ des exportations de la capitale en $1860^{5}$.

Engagée dans une recherche perpétuelle de nouveautés, cette industrie, «mobile» par sa force de proposition, fonctionne largement sur la capacité à lancer des modes. «Nulle part on est plus habile à exciter la consommation par mille séductions, à pourvoir par avance à des caprices ou à des besoins auxquels on donne ainsi naissance. [...] Les saisons, les modes, les circonstances, font modifier la façon et la matière de bien des objets usuels et le monde élégant de tous les pays attend chaque année de Paris les nouveautés en tous les genres ${ }^{6} »$, précise un membre du jury central de France de l'Exposition universelle de 1867.

Encore faut-il préciser que chacun de ces produits est décliné en des gammes de prix souvent très étendues, témoignant de l'emploi de matières différentes en factures plus ou moins fines. Une part de la spécificité des articles de Paris réside dans cette capacité à toucher des pouvoirs d'achats différents.

Dans ce cadre, le jeu sur la matière est la règle. En matière noble, en matière dégradée, ou en matières innovantes jouant sur l'imitation, les articles de Paris sont déclinés en de multiples compositions. Dans le secteur de la tabletterie ${ }^{7}$ travaillant l'ivoire, l'écaille, la nacre, l'os, la corne ou le coco, la diversité des objets fabriqués tient des plus belles matières ou de leurs rebuts quand elle ne réside pas dans l'usage de matières "synthétiques" telles que le permet l'essor de certaines gélatines à l'imitation de nacre et d'écaille.

Si une part de la qualité reconnue réside dans la matière mise en œuvre, ou, en tout cas, dans le discours qu'elle autorise pour faire la promotion des articles, la facture est également essentielle. Il s'agit de distinguer entre une facture qui renvoie à la solidité de l'objet, l'efficacité de son service, sa commodité, et celle qui relève d'un argumentaire des qualités du fabricant: le goût et l'imagination.

\section{Le «bon goût » des articles}

L'art, la mode, la fantaisie, l'élégance, le cachet et surtout le goût forment le cœur du vocabulaire des discours sur la qualité des articles de Paris au XIX $x^{e}$ siècle.

S'il détermine la «manière dont une chose est faite, caractère particulier de quelques ouvrages ${ }^{8} »$, le goût définit également une "faculté toute spontanée qui précède la réflexion que tout le 
monde possède mais qui est différent chez chacun ${ }^{9}$ ». Le concept apparaît plastique car il renvoie autant à l'offre qu'à la capacité presque naturelle qu'un individu a à apprécier un objet. Il s'agirait par conséquent d'une donnée préexistante, relative et intrinsèque à la demande, tout en étant également selon «la faculté acquise ou innée qui nous fait discerner l'à propos, la convenance de telle ou telle chose, de tel ou tel genre, de telle ou telle manière ${ }^{10} »$. Ce goût, cette faculté s'élabore dans une offre toujours renouvelée de produits qui semble se fixer sur une référence: «le bon goût». Celle-ci exprime un rapport à la modestie, à l'absence d'ostentation; le style, l'élégance se départissent $\mathrm{du}$ faste des matières au cours du XIx ${ }^{e}$ siècle. Le discours justifie la dégradation de la qualité qui fait place à un art de vie bourgeois. «Il y aura toujours une place à part dans les préférences des gens de goût, je veux dire de ceux qui font le plus cas de l'art que de la matière, et qui aiment mieux un bronze finement sculpté qu'un bijou $\mathrm{d}^{\prime}$ or accusant un travail inférieur ${ }^{11}$.» $\mathrm{Au} \mathrm{XIX}^{\mathrm{e}}$ siècle, les qualités des "articles de Paris», définies par une matière, une facture et une esthétique, renseignent sur les changements de consommation de la société autant que sur les stratégies mises au point par les fabricants et les marchands pour élargir leur clientèle. Elles semblent attester l'apparition d'une «monoculture» dont l'influence s'étendrait dans la seconde moitié du siècle ${ }^{12}$.

«Si l'on remontait à l'origine de la création de bien des objets élégants de cette industrie française si estimée dans le monde entier, l'on verrait que leur valeur est souvent due à l'imagination d'une simple ouvrière, qui a eu le goût assez pur pour créer heureusement une forme nouvelle ${ }^{13}$.» Cette appréciation de la qualité des articles de Paris et plus globalement des fabrications françaises fait du lien entre art et savoir-faire un facteur explicatif de leur notoriété.

L'éclatement des qualités observé dans les consommations de la bourgeoisie française conditionne une part de l'offre à l'exportation, notamment à travers la promotion d'un certain goût français relevant largement d'un accord entre art et industrie ${ }^{14}$.

La référence à l'art, autre composante du discours sur la qualité des articles de Paris, semble devenir une clé de compréhension de ce «bon goût» dont se revendiquent les fabricants français dans leur appréciation de leurs offres.

Ce rapport à l'art, valorisé dans le discours des fabricants, trouve-t-il sa justification dans la compétence des ouvriers?

\section{La fée aux mille doigts}

\section{Des doigts habiles?}

À partir des déclarations de 6124 entrepreneurs faites lors de l'enquête de la Chambre de commerce et d'industrie de Paris en 1847-1848, il est possible de dénombrer les forces engagées dans ces fabrications d'articles de Paris. Avant la crise de 1848, la fabrication des articles de Paris emploie 35679 ouvriers sur les 
342530 employés à Paris. Onze ans plus tard, la seconde enquête, reposant sur une nomenclature quelque peu différente, signale une tendance à la baisse.

Effectifs travaillant à la fabrication d'articles de Paris 1847/1860

\begin{tabular}{|c|c|c|c|c|}
\hline $1847 / \mathbf{1 8 6 0}$ & Hommes & Femmes & $\begin{array}{c}\text { Enfants } \\
\text { et jeunes gens }\end{array}$ & Totaux \\
\hline Articles de Paris & $17583 / \mathbf{1 0 7 4 2}$ & $15540 / \mathbf{1 2 6 1 9}$ & $2556 / \mathbf{2 3 3 7}$ & $\begin{array}{c}35679 / \\
\mathbf{2 5 6 9 8}\end{array}$ \\
\hline Totaux & 204925 & 112891 & 24714 & 342530 \\
& $/ \mathbf{5 5 0 2 8 0}$ & $/ \mathbf{1 0 5 4 1 0}$ & $/ \mathbf{2 5 5 4 0}$ & $/ \mathbf{4 1 6 8 1 1}$ \\
\hline
\end{tabular}

Rapportées aux secteurs d'activité, les deux enquêtes montrent une certaine stabilité dans l'importance des emplois offerts par secteur, la hiérarchie des secteurs en fonction de leur taux d'emplois demeure similaire si l'on homogénéise la classification «articles de Paris» retenue.

Effectifs en fonction des secteurs de la fabrique de Paris, 1847 / 1860

\begin{tabular}{|l|r|r|}
\cline { 2 - 3 } \multicolumn{1}{c|}{} & 1847 & 1860 \\
\hline Fleurs artificielles & 6771 & 6266 \\
\hline Cartonnage & 2530 & 1245 \\
\hline Boutons métal et tissus & 1446 & 1114 \\
\hline Bimbeloterie & 2469 & 796 \\
\hline Plumassiers & 718 & 671 \\
\hline Eventails & 684 & 659 \\
\hline Parapluies et ombrelles & 1796 & 631 \\
\hline
\end{tabular}

La proportion de femmes passe de $43,5 \%$ à $49 \%$ : les femmes sont employées majoritairement chez les fabricants de fleurs artificielles et chez les plumassiers, chez les fabricants de cartonnage et dans les éventails. Le niveau d'instruction des femmes et des hommes engagés dans ces secteurs apparait relativement bon, puisque seulement $6 \%$ ne savent ni lire ni écrire. Parallèlement, la proportion de jeunes de moins de 16 ans représente respectivement $7 \%$ puis $9 \%$ des effectifs employés dans les industries rattachées aux articles de Paris.

En 1848, le détail de l'enquête permet de saisir leur répartition: les apprentis sont surtout nombreux chez les fleuristes, il y a en 664 , chez les tabletiers où l'on en trouve 168, et 224 dans la fabrication du cartonnage. Dans cette dernière industrie, la proportion est d'un apprenti pour six ou sept ouvriers. Dans certains secteurs comme la fabrique de boutons, les très nombreux enfants ne sont pas considérés comme des apprentis mais comme des ouvriers. Ainsi, la fabrication des boutons de corne, d'os ou de nacre comprend seulement 10 apprentis engagés par contrat contre 101 jeunes ouvriers salariés. Le niveau d'instruction révélé par les enquêtes montre que $95 \%$ des hommes et $83 \%$ des femmes savent lire et écrire.

Les compétences ou la certification des ouvriers ne sont que peu abordées dans ces enquêtes. Pour les saisir, il importe de comprendre les spécificités de secteurs qui s'inscrivent dans des conditions de travail déterminant une part des qualités attendues de la main-d'œuvre. 


\section{Conditions de travail}

En classant sous une même rubrique des articles fort divers, les contemporains ont souhaitéregrouper sous le terme générique $\mathrm{d}^{\prime}$ «articles de Paris» des produits dont la fabrication recouvre les mêmes lignes de force: une extrême division du travail, une forme de spécialisation, un rythme saisonnier. L'organisation d'une partie de la fabrique de Paris s'inscrit autant dans un travail en chambre que dans un travail en atelier.

Par trois ou quatre dans de petits ateliers, rarement au-delà de dix ou douze réunis, les ouvriers fabriquent souvent en famille sur la base d'une division du travail. Cette division des tâches, associée à un rythme rapide, renvoie au problème de l'aliénation des ouvriers posé par l'économie politique. Dès 1823, Heinrich nomie politique ${ }^{15}$, puis, dans les années 1840, Eugène Buret ${ }^{16}$, élève de Sismondi, font de la division du travail une cause explicative de la misère des populations. Réduit à une «force de travail» ou pour le moins à une "adresse de l'habitude», l'ouvrier ne disposerait plus de capacités créatives et perdrait une part de l'habilité des doigts. Cette appréciation rejoint en partie celle faite lors de l'enquête sur la condition ouvrière à Paris en 1880. Deux secteurs témoigneraient de l'absence de qualification ou de sa perte dans la fabrication des articles de Paris.

La bijouterie en faux avec une forte mécanisation devient progressivement au cours du siècle un espace d'assemblage de pièces estampées. Piel, fabricant de bijouterie en imitation, explique la perte de savoir-faire professionnel dans les années 1880: «On emploie

aujourd'hui dans la bijouterie une grande quantité d'apprêts, ce qui diminue singulièrement la difficulté et la perfection du travail. Les ouvriers qui arrivent à souder très vivement toutes les pièces apprêtées, estampées, gagnent bien leur vie ${ }^{17}$.»

La simplification de la fabrication par le recours à la préparation mécanique des pièces conduit à une déqualification des tâches dans la mesure où les ouvriers ne procèdent plus qu'à de simples emboîtages $^{18}$. Les fleurs artificielles, le secteur le plus important de la fabrique d'articles de Paris, semblent également connaitre une division du travail extrême, au point d'être jugées comme une source de l'appauvrissement de la créativité du secteur à la fin du XIXe siècle. Ici, la tendance apparait quelque peu différente. Si la division du travail est forte, de l'apprêt à la découpe en passant par la teinture, l'ouvrière est spécialisée dans une seule variété de fleurs, tandis que le montage demeure du ressort de la seule monteuse.

Poser en ces termes la question de la spécialisation revient à souligner une forme d'incapacité à faire autrement ou autre chose qui, si elle ne sclérose pas la fabrication, conditionne au moins les modèles.

«La division du travail, nécessitée par les besoins d'une production excessive et à bon marché, l'emploi des procédés mécaniques qui a facilité et justifié cette division sont des faits d'ordre général qui ont partout porté atteinte à l'initiative de l'ouvrier, diminué sa responsabilité et par suite affaibli son savoir ${ }^{19}{ }^{\prime}$, constate Antonin Proust en ouvrant les travaux de la commission d'enquête sur la situation des ouvriers et des industries d'art. Cette forte division du travail, si elle est perçue comme une cause de l'aliénation 
de l'esprit créatif de l'ouvrier, trouve sa justification dans le rythme de production de ce type de produits. Parce qu'elles sont des effets de modes, inscrits dans une saisonnalité, les fabrications doivent être faites rapidement pour répondre notamment au calendrier commercial. Aussi est-il intéressant de noter que la rapidité d'exécution est une compétence précieuse revendiquée dans le discours des fabricants.

«Ces ouvriers se soumettent avec une merveilleuse facilité à toutes les exigences des consommateurs, [...] ont l'esprit inventif, ils ont le travail actif mais intermittent, ils vont à la besogne comme, dans d'autres circonstances, ils monteraient à l'assaut avec l'énergie après laquelle il leur faut pouvoir prendre un long repos. [...] On obtient facilement de la fabrique parisienne des efforts incroyables de production, les nuits sont employées au travail et chacun semble n'avoir d'autre préoccupation que celle de terminer sa tâche ${ }^{20}$. »

\section{La formation en question}

\section{Art et industrie}

La question de la formation prise dans son acception large invite à saisir comment elle fut pensée pour développer l'offre et faire face à la concurrence internationale.

La réforme de l'enseignement des arts industriels est affirmée dans le rapport du comte Léon de Laborde en 1851, avant que Prosper Mérimée n'alerte le gouvernement français sur la nécessité de renforcer le lien entre art et industries, pour affirmer
La fabrication des articles de Paris s'inscrit donc dans une flexibilité du travail dictée par le rythme de commercialisation, une rapidité d'exécution, qui implique une division du travail et une mécanisation.

Derrière les discours faisant la promotion du goût, de l'élégance, du cachet des produits, les compétences ou qualifications des ouvriers engagés reposent sur une réalité quelque peu différente. L'habileté dans la reproduction des gestes, la spécialisation dans un type de fabrication, la flexibilité dans le rythme de la production sont des qualités recherchées qui font le bon ouvrier. Dans ce cadre, l'apprentissage est important puisqu'il permet autant d'acclimater le jeune à ces conditions de travail que de fixer les manières de faire. La question de la formation et surtout du rapport à l'art est cependant une nécessité qui semble avoir été une préoccupation récurrente des fabricants.

le goût français sur la concurrence ${ }^{21}$. La création de l'Union centrale des beaux-arts appliqués à l'industrie est une première réponse, l'amélioration des formations en arts appliqués en référence à celles proposées dans les manufactures royales d'Ancien Régime en est une deuxième.

Mais, si le cap est fixé, la question du lien entre la théorie et la pratique, entre le beau et l'utile, c'est-à-dire entre l'art et l'industrie, pose problème.

En 1857, à la suite d'une enquête sur les branches du mobilier et de l'ébénis- 
terie, Émile Reiber, architecte, ne peut que constater l'ignorance des patrons et des apprentis en matière $d^{\prime}$ art, tout en signalant une déviance commune née de l'essor de collaborations avec des artistes: "Le patron par suite de cette collaboration émet des prétentions artistiques et il se fait volontiers décerner le titre d'artiste. S'il ne se fait pas donner ce titre par l'assentiment de ses contemporains, il y a une certaine presse qui moyennant conditions se met à ses ordres et le public peut lire dans des articles réclames que $M$. un tel (qui ne sait pas tenir un crayon) est un très grand Artiste ${ }^{22} . »$

La critique tranche singulièrement avec les discours des rapports des expositions universelles qui accordent aux articles de Paris une élégance née justement d'une expression artistique maîtrisée. Ce constat rend compte de l'intérêt «promotionnel» que représente le rattachement de l'industrie à l'art. Rapporté aux articles de Paris, qui misent sur des créations esthétiques séduisantes rapidement renouvelées, le lien entre art et industrie gagne en pertinence. L'apprentissage est la règle, mais il devient progressivement difficile de conserver une main-d'œuvre en formation qui trouve des engagements plus lucratifs dans des fabrications d'articles de bas de gamme. Cette constante s'affirme dans les années 1870. L'élargissement des consommations de petits articles de bas prix conduit à un avilissement de la formation pour des produits de meilleure facture. La mode pousse au renouvellement rapide et se départit de la qualité de fabrication. Aussi, des apprentis en cours ou en fin de formation trouvent-ils plus d'intérêt à fabriquer chez des petits façonniers des produits de piètre qualité contre un salaire d'emblée supérieur.

La proximité de certains savoir-faire est aussi un problème pour conserver les apprentis dans leur cœur de métier. Ainsi, Madame Guérin Brécheux, fabricante d'éventails, reconnaît qu'une part de ses ouvrières provient de nombreuses industries. «La porcelaine, le papier-peint, les émaux nous procurent les peintres, les petits meubles, les vernisseurs, les décorateurs. Quant aux brodeuses, plumassières, monteuses, passementières, nos salaires qui sont relativement élevés nous assurent autant de mains que nous en pouvons utiliser ${ }^{23}$.»

La perméabilité entre secteurs est aussi la règle dans le travail de l'ivoire, des écailles, des os ou des bois. C'est un aspect de la mobilité des apprentis et des ouvriers qui nous semble être un point essentiel de la formation indirecte acquise en atelier, une sorte de formation continue autorisée par un socle de connaissances pratiques acquises par le passage dans divers ateliers.

\section{Publiques ou privées : des initiatives variées}

La formation des ouvriers a fait l'objet d'initiatives publiques comme privées tant est constant le souci des contemporains d'améliorer les compétences artistiques des fabricants considérant que «l'art est l'âme de l'industrie ${ }^{24} »$.

L'action de l'État, malgré de nombreuses initiatives, est demeurée assez limitée. Après 1830, Charles Dupin, le ministre de l'Intérieur, institua une commission chargée de rechercher les moyens de venir en aide aux industries, 
qui recommandait l'enseignement obligatoire du dessin dans tous les établissements scolaires. L'idée fut reprise et réaffirmée sans suite par le gouvernement en 1845 et après chaque exposition universelle de 1851 à 1878. Dans ces projets, l'apprentissage du dessin dispose d'une place centrale. Ainsi, par les arrêtés ministériels de 1878 instituant l'enseignement obligatoire du dessin dans les établissements publics d'enseignement primaire et secondaire, la formation du dessin gagne du terrain. Les progrès sont cependant encore médiocres et cela malgré l'initiative des membres du parlement, de la ville de Paris, des départements pour établir les bases de l'organisation de l'enseignement des arts, dont le point de départ est l'enseignement du dessin. La loi sur les écoles manuelles d'apprentissage du 11 décembre 1880 devait régler une part de l'enseignement manuel, tandis que la reconnaissance légale des syndicats professionnels permettrait de développer des écoles spéciales à chacune des industries ou même des écoles donnant une instruction commune à plusieurs industries similaires.

Plusieurs secteurs relevant des articles de Paris semblent avoir été particulièrement innovants pour développer un enseignement professionnel.

Tel est le cas de l'école d'apprentissage en fleurs artificielles, créée par le comité de patronage de la Chambre syndicale des fleurs, plumes et modes, sous la direction de Charles Petit, en 1870. La formation pratique dispensée en atelier s'organise ainsi: «Nous voulons que les jeunes apprenties sachent faire depuis le commencement jusqu'à la fin dans la fabrication des roses, en y comprenant l'art d'obtenir les nuances. Ordinairement ce sont des ouvriers spéciaux qui font le trempage des roses, ce que nous appelons des trempeurs. Nous insistons pour que les jeunes filles apprennent aussi à tremper. Si elles ont du goût, les apprenties peuvent, après trois ou quatre ans de travail, devenir de bonnes ouvrières et même s'établir, faire leurs nuances elles-mêmes et n'avoir pas besoin de recourir au trempeur qui coûte fort cher ${ }^{25}$.»

Posé ainsi, l'objectif de la formation est non seulement de former des ouvrières, mais aussi de futures entrepreneuses. Si la diversification de la maitrise des techniques de fabrication de fleurs est de mise, l'apprenti se spécialise sur la fleur la plus difficile, la rose au titre du précepte: "Quand on sait le plus on sait le moins $^{26}$.»

«L'école de dessin de la bijouterie et des industries qui s'y rattachent $»$ ouverte par la chambre syndicale de ce métier en 1876 offre un autre exemple singulier des formations en vigueur dans les secteurs de fabrication des articles de Paris. Cette école s'adresse à des apprentis engagés dans ce secteur, mais aussi aux patrons désireux d'acquérir des connaissances en matière de dessin et de modelage. Rapidement, la formation glisse de la maîtrise du dessin et du modelage à la réalisation de pièces exécutées en atelier. La formation fait de l'organisation coup double en introduisant l'art dans l'atelier par les petites mains de l'apprenti, «l'apprenti est le meilleur coursier de ma propagande. Avec l'apprenti j'améliore l'éducation artistique du patron et celle de l'ouvrier ${ }^{27}$.»

Si le savoir technique et la pratique s'enseignent, l'habilité, la dextérité de la 
main s'acquièrent à force d'expérience. La reconnaissance se gagne alors dans des concours organisés pour récompenser et distinguer les meilleurs apprentis. C'est un fait commun à ces écoles d'apprentissage, à ces patronages, de participer à la promotion de leurs élèves en exposant leurs produits. Il en va, semble-t-il, de la reconnaissance du goût dont ils témoignent. Cette volonté trouve une dimension particulière rapportée aux articles de Paris.

\section{Vers la composition d'une identité de place}

La question de la formation des ouvriers parisiens devient un problème récurrent dans la seconde moitié du $\mathrm{XIX}^{\mathrm{e}}$ siècle, au moment où le ralentissement des affaires se fait sentir. Dès lors s'affirme un discours sur le goût, l'élégance et la fantaisie des produits, justifiés par les compétences inégalées des ouvriers de la capitale.

«Le développement très populaire des beaux-arts, les nombreuses applications qui sont journellement faites des sciences à l'industrie, le voisinage, le contact, la relation de tant de travaux divers, forment comme un ensemble d'enseignements qui pénètre à son insu, toute la population industrieuse, et qui agit, indépendamment de l'instruction offerte dans les écoles, sur tous les enfants de Paris. Il en résulte une grande précocité et une grande vivacité d'intelligence chez les travailleurs ${ }^{28}$.»

À travers les rapports, les enquêtes menées par des fabricants, la créativité et le goût des ouvriers parisiens sont des références fortes pour revendiquer la suprématie de l'offre parisienne. Cette association de la ville et du savoir-faire apparaît d'autant plus importante que les ouvriers de la fabrique disposeraient d'une bonne qualification du fait de leur implantation à Paris. S'il paraît exagéré, l'argument développé trouve une part de sa justification dans le processus de renouvellement des articles de Paris. Procédant de proche en proche, en jouant sur l'imitation, la réadaptation, l'emprunt ou le détournement, une part de la fabrication parisienne repose sur sa capacité à digérer rapidement les nouveaux produits offerts par la capitale. Dans ce cadre, il apparaît que la défense et la promotion de l'image de Paris passent par ce lien entre les compétences des ouvriers et leur fabrication. Il s'agit d'affirmer et de promouvoir une identité de place qui fait de Paris à la fois le lieu de création et d'innovation de produits nouveaux grâce à l'ingéniosité de ses ouvriers ainsi que le creuset de nouvelles modes reprises à l'étranger.

\section{Notes}

1. Nadège Sougy est professeure assistante $\mathrm{d}$ 'histoire moderne et contemporaine à l'université de Neuchâtel. Ses travaux portent sur les qualités et les certifications des produits manufacturés et des objets de luxe aux XVIII $^{\mathrm{e}}$ et $\mathrm{XIX}^{\mathrm{e}}$ siècles. Elle s'intéresse notamment à la bijouterie, l'orfèvrerie et l'horlogerie en France et en Suisse. Elle a dirigé et publié les actes du colloque Luxes et internationalisation (Xvie-XIxe siècles) (Alphil, 2013). Contact: nadege.sougy@unine.ch

2. La Fabrique de Paris. Moniteur du commissionnaire et de l'exportateur, $\mathrm{n}^{\circ} 1$, jeudi 29 mai 1862, p. 1 .

3. Ibid., p. 1. 
4. Gérard Gayot, Philippe Minard (dir.), «Les ouvriers qualifiés de l'industrie (xvie-xxe siècles). Formation, emploi, migrations», Revue du Nord, hors série, 2001.

5. L'exportation s'élève à $88 \%$ pour les éventails, les nécessaires, $41 \%$, les fleurs artificielles, $33 \%$, les boutons en métal et tissu, $30 \%$, Statistique de l'industrie à Paris résultant de l'Enquête faite par la chambre de commerce, 1864, p. XLVII.

6. Nathalis Rondot, Exposition universelle de 1862, Paris, 1863, p. 7.

7. Nadège Sougy, «D'écaille, de nacre ou d'ivoire: les matières du désir au XIX ${ }^{\mathrm{e}}$ siècle", dans Natacha Coquery et Alain Bonnet (dir.), Le commerce du luxe, le luxe du commerce. Production, exposition et circulation des objets précieux du Moyen Âge à nos jours, Paris, Mare \& Martin, 2014.

8. Émile Littré, Dictionnaire de la langue française, vol. 2, Paris, 1873-1874, p. 1902.

9. Ibid.

10. Ibid.

11. Louis Enault, Les arts industriels: Vienne, Londres, Paris, Paris, , 1877, p. 50.

12. Manuel Charpy, "L'ordre des choses. Sur quelques traits de la culture matérielle bourgeoise parisienne, 1830-1914», Revue d'histoire du XIX siècle, 2007/1, n 34, p. 105-128.

13. Charles Laboulaye, L'art industriel ou les beaux-arts considérés dans leurs rapports avec l'industrie moderne, Paris, 1887, p. 6.

14. Nadège Sougy, "Du beau et de l'utile: les qualités des fabrications industrielles (18401870)", dans Pierre Lamard et Nicolas Stoskopf (dir.), Art $\mathcal{E}$ industrie xvIIIe-XxIe siècle, Paris, Picard, 2013, p. 25-37.

15. Heinrich Friedrich Storch, Cours d'économie politique ou Exposition des principes qui déterminent la prospérité des nations, t. III, Paris, 1823, p. 339.
16. Eugène Buret, De la misère des classes laborieuses en Angleterre et en France: de la nature de la misère, de son existence, de ses effets, de ses causes, et..., vol. 2, Paris, 1840.

17. Commission d'enquête sur la situation des ouvriers et des industries d'art, instituée par décret en date du 24 septembre 1881, Paris, 1884, p. 90.

18. Nadège Sougy, "Assembler pour fabriquer: la question de l'interchangeabilité des pièces", catalogue d'exposition, La Chaux-deFonds, Éditions Institut L'homme et le temps, 2011, p. 132-140.

19. Commission d'enquête sur la situation des ouvriers..., op. cit., p. XXVI.

20. Ibid.

21. Sur le développement de l'enseignement des arts appliqués en France et sur le rapport du comte de Laborde sur les industries d'art rédigé au nom de la Commission française du Jury international de l'Exposition universelle de Londres en 1851, Stéphane Laurent, Les arts appliqués en France. Genèse d'un enseignement, Paris, CTHS, 1999.

22. Commission d'enquête sur la situation des ouvriers..., op. cit., p. 246.

23. Ibid., p. 337.

24. Ibid., p. VIII.

25. Ibid., p. 167.

26. Ibid.

27. Ibid., p. 255.

28. Chambre de commerce et d'industrie de Paris, Statistique de l'industrie à Paris: résultant de l'enquête faite par la chambre de commerce pour les années 1847-1848, 1851, p. 61-62. 